Revue des patrimoines

$29 \mid 2016$

Ensembles mobiliers, industriels, techniques.

Connaissance, protection, conservation, présentation au public

\title{
Enquête sur l'environnement socio-technique d'un générateur Cockcroft-Walton en vue de sa restauration et de sa conservation
}

Research on the social and technical environment of a Cockcroft-Walton

generator prior to its restoration and conservation

Rémy Geindreau, Séverine Derolez et Jean-Paul Martin

\section{(2) OpenEdition}

\section{Journals}

Édition électronique

URL : http://journals.openedition.org/insitu/12935

DOI : 10.4000/insitu. 12935

ISSN : 1630-7305

Éditeur

Ministère de la culture

Référence électronique

Rémy Geindreau, Séverine Derolez et Jean-Paul Martin, «Enquête sur l'environnement socio-technique d'un générateur Cockcroft-Walton en vue de sa restauration et de sa conservation », In Situ [En ligne], 29 | 2016, mis en ligne le 13 juillet 2016, consulté le 03 mai 2019. URL : http:// journals.openedition.org/insitu/12935; DOI : 10.4000/insitu.12935

Ce document a été généré automatiquement le 3 mai 2019.

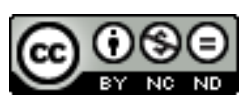

In Situ Revues des patrimoines est mis à disposition selon les termes de la licence Creative Commons Attribution - Pas d'Utilisation Commerciale - Pas de Modification 4.0 International. 


\title{
Enquête sur l'environnement socio- technique d'un générateur Cockcroft-Walton en vue de sa restauration et de sa conservation
}

\author{
Research on the social and technical environment of a Cockcroft-Walton \\ generator prior to its restoration and conservation
}

Rémy Geindreau, Séverine Derolez et Jean-Paul Martin

\section{Introduction}

L'expérimentation, dans cette branche de la science, est coûteuse. Il n'est plus possible de se contenter de pièces détachées empruntées aux collections de cabinets de physique. [...] Nous ne sommes plus au temps de Fresnel où les découvertes importantes en optique ont pu être faites avec un carton percé et une goutte de miel ! Jean Thibaud ${ }^{1}, 1954$.

1 Le progrès accéléré des connaissances scientifiques et les prouesses expérimentales réalisées au siècle dernier se sont appuyés sur la réalisation d'instruments de plus en plus performants. La course aux hautes énergies ${ }^{2}$ nécessaire à l'accélération des particules dans le domaine de la physique nucléaire a notamment conduit les laboratoires à se doter d'appareillages lourds et coûteux. L'évolution des accélérateurs de particules, devenus objets d'investigation en eux-mêmes, en témoigne: du cyclotron d'Ernest Lawrence (1931), des générateurs Van de Graaff et Cockcroft-Walton (1932) jusqu'à aujourd'hui avec le LHC (Large Hadron Collider) du CERN ${ }^{3}$ dont la circonférence se mesure en kilomètre.

Dans le même temps, le patrimoine s'ouvre à de nouveaux objets dont ceux de la science de la deuxième moitié du $\mathrm{XX}^{\mathrm{e}}$ siècle et notamment ceux des établissements universitaires et de recherche en physique nucléaire. Ainsi, les premiers accélérateurs devenus obsolètes sont depuis peu candidats à la patrimonialisation. On note, par exemple, la 
classification au titre des monuments historiques (2007) d'un proche cousin de l'objet que nous avons étudié, le générateur d'impulsion dit «l'éclateur » utilisé par les Joliot-Curie au laboratoire d'Ivry'.

3 Au début des années 1950, le générateur Cockcroft-Walton ${ }^{5}$ (fig. 1) de l'Institut de physique nucléaire de Lyon (IPNL) 6 a été acheté par le physicien français Jean Thibaud (1901-1960) à la société Haefely pour 20 millions de francs, grâce à une aide de l'Armée de terre. D'un point de vue utilitaire, il s'agit d'un appareil qui multiplie et redresse la tension pour fournir une énergie suffisamment grande pour accélérer les particules dans un tube accélérateur. Ce «briseur d'atomes » fut utilisé à Lyon à la caserne militaire de la Vitriolerie (de 1953 au début des années 1960) puis à l'IPNL (à partir de 1962) où il est actuellement entreposé. Il est typique de ce que les historiens nomment la "Big Science ", née pendant et après la Seconde Guerre mondiale lorsque l'industrie, les États, l'économie, le militaire et l'ingénierie vont s'associer pour le financement et l'organisation de projets de recherches scientifiques de grande envergure. Les laboratoires désormais à dimension industrielle vont alors se doter d'instruments sophistiqués devenus indispensables à l'étude des particules subatomiques. Dépassé par des accélérateurs de particules plus performants, démonté depuis la fin des années 1990, le générateur Cockcroft-Walton a fait l'objet de deux projets muséographiques avortés. Les tubes accélérateurs reliés au générateur ont été détruit ainsi que la plupart des éléments (pupitres, appareils, etc.) qui composait cet ensemble. Le générateur est alors entreposé en pièces détachées durant une dizaine d'année dans un laboratoire toujours en activité. 
Figure 1

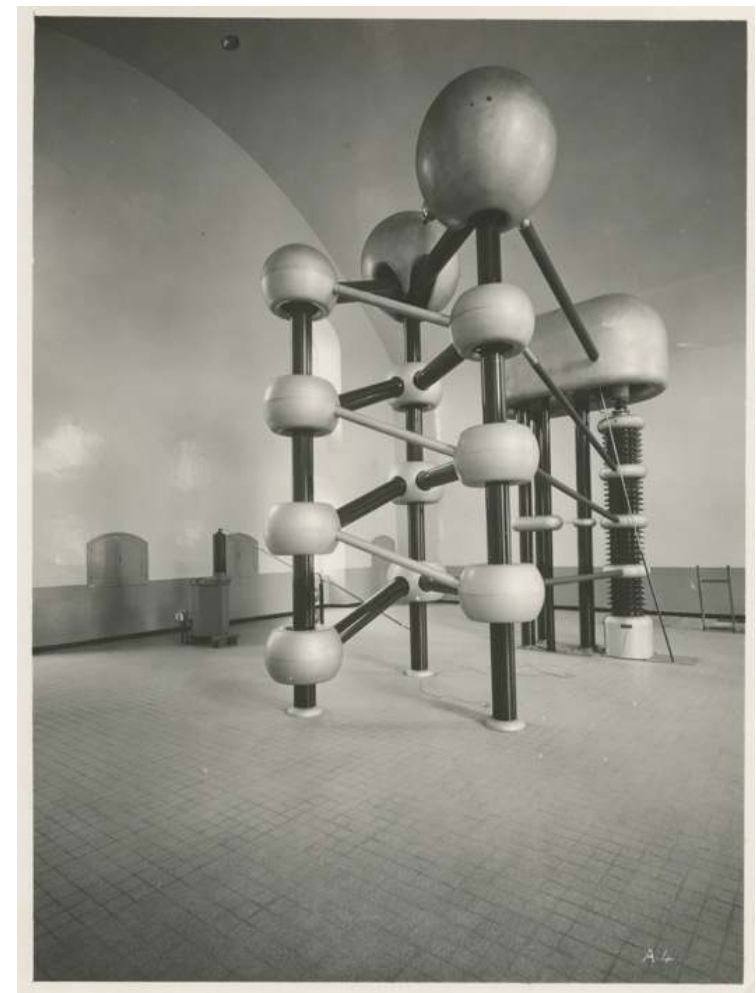

LE gÉNÉRATEUR COCKCROFT-WALTON AU FORT DE LA VITRIOLERIE AVEC UN TUBE ACCÉLÉRATEUR 1,2 MÉgA-ÉLECTRONS-VOLTS, UN TRANSFORMATEUR ET DEUX ÉCLATEURS. ON DISTINGUE UNE ÉCHELLE PERMETTANT DE REJOINDRE L'ÉTAgE INFÉRIEUR OÙ SE TROUVENT LES CIBLES.

Phot. IPNL. @ IPNL, Institut de physique nucléaire de Lyon, 1953.

Aujourd'hui, un nouveau projet de valorisation émerge des efforts conjoints de différents acteurs de l'université Claude Bernard à Lyon. Depuis 2012, un physicien CNRS/IPNL, une doctorante du laboratoire S2HEP (Science et Société, Historicité, Éducation et Pratiques), le responsable des collections de physique de l'université ainsi que le responsable du département de physique se sont fixé pour objectif de conserver, remonter et valoriser le générateur. Différentes actions ont été engagées, comme celle de faire appel aux compétences d'un élève du département de conservation-restauration de l'école supérieure d'art d'Avignon. Ainsi, Rémy Geindreau a consacré son mémoire de fin d'études à la conservation-restauration de cet objet ${ }^{7}$. Le travail a particulièrement porté sur les conditions de conservation nécessaires au respect de l'intégrité matérielle et de l'identité patrimoniale de cet objet. Le choix d'un lieu de conservation est une composante principale du futur projet de remontage: sous quelles conditions est-il vraiment possible de remonter cet objet? Comment assurer la survie de cet ensemble technico-scientifique obsolète et fragmenté dans la communauté qui l'a produit?

\section{Les défis de la sauvegarde des grands instruments du $\mathrm{XX}$ siècle}

5 Cet objet, qui culmine à $7 \mathrm{~m}$ de haut, et dont quelques rares exemplaires sont exposés dans le monde, pose de nombreux défis qu'il nous a fallu relever. En effet, sortir d'un 
laboratoire une machine en pièces détachées, faire de sa particularité technique et historique et de la crainte de sa disparition la raison de sa conservation, rompre le réseau sociotechnique dont elle était solidaire sont des actions qui ont des incidences sur le projet de préservation. De plus, insérer le générateur dans le champ patrimonial, le documenter, le remonter, suppléer à la destruction des appareillages associés, accorder une équipe pluridisciplinaire, traiter les matériaux récents peu abordés en conservationrestauration (alliage d'aluminium, polymères, etc.), l'exposer inerte ou non, in situ ou ex situ, réunir les fonds nécessaires pour le coût des opérations et enfin, tenter de maintenir en dépit de ses métamorphoses, l'authenticité supposée de l'objet: toutes ces actions, nous le verrons, soulèvent des difficultés, en théorie comme en pratique.

\section{Un objet hors normes}

6 Comme la plupart des objets scientifiques et techniques, cet objet n'est ni rare ni unique, puisque produit industriellement en plusieurs exemplaires. Il correspond aux nouveaux modes de fabrication de l'instrumentation électrique qui change avec l'avènement des techno-sciences. C'est ce que relève notamment Dominique Pestre :

Second changement majeur apporté par la guerre de 1940 et la guerre froide, celui concernant la culture du milieu - les outils et appareillages quotidiennement utilisés dans les laboratoires, aussi bien que les manières de traiter des questions. Ces équipements sont rendus méconnaissables par deux ou trois décennies de développements technologiques intensifs et tous azimuts ${ }^{8}$.

7 Cela dit, le générateur Cockcroft-Walton, emblématique de ces transformations, peut être considéré comme « hors normes » pour plusieurs raisons : sa taille, son poids, sa fonction passée, son lieu de conservation et sa destination, son esthétique, la nature de ses matériaux, son domaine d'application, son obsolescence, son état de conservation (en pièces détachées avec une documentation technique disséminée ou disparue) et son statut.

8 Si intégrer un tel objet dans un musée constitue en quelque sorte un fantasme de conservateur, les grandes dimensions et le poids de l'objet sont des obstacles à sa sauvegarde. Ce gigantisme impose des contraintes considérables, sinon herculéennes, du point de vue de l'étude préalable comme de celui de la réalisation des traitements (infrastructures lourdes, constat et diagnostic complexes, etc.). L'exposition de ces objets est souvent reléguée à l'extérieur, ce qui n'est pas, comme nous le verrons, sans risque pour leur conservation comme pour leur identité. Enfin, du fait que son lieu de stockage soit un laboratoire en activité, son accessibilité dépend des règles de sécurité du bâtiment, de ses heures ouvrables, des plannings de manipulation et de la disponibilité des chercheurs pour accueillir des visiteurs. Cette contrainte est d'ailleurs généralisable à de nombreux gros objets du patrimoine universitaire qui, faute de lieu de stockage ou de conservation, s'ils ne sont pas détruits, resteront dans les locaux utilisés par les chercheurs. S'ajoutent à cela des précautions sanitaires indispensables liées aux éventuels risques de radioactivité ou de présence de produits toxiques. Notons que ces contrôles d'innocuité, effectués par des professionnels de la radio-protection, sont soumis à une réglementation', et qu'il s'agit de mesures d'hygiène obligatoires pour toutes les personnes, public compris, amenées à se tenir au plus près des rebuts de la physique.

9 Le patrimoine scientifique contemporain se distingue aussi par la nature des matériaux qui le compose. Les matériaux dits « précieux » tels que le bois, l'ivoire, le laiton, l'ébène 
ont été délaissés au profit d'alliages (ici, alliage d'aluminium au silicium), de polymères, de composés électroniques offrant des capacités techniques plus larges mais aussi plus complexes. Dans certains cas, la composition d'un matériau peut même être soumise à des brevets, et des analyses scientifiques sont nécessaires afin d'en connaitre précisément la composition. Par exemple, le générateur comprend des redresseurs, condensateurs, résistances et isolateurs en résine phénol-formaldéhyde ( $\mathrm{PF}$ de la famille des phénoplastes), choisie pour sa résistance mécanique et électrique et sa tenue à la chaleur. Le directeur de l'entreprise de l'époque (M. Métraux) parle de «cylindre en papier bakélisé ", de même, nous savons que le fabricant Emil Haefely a breveté une matière plastique dite haefelyte ${ }^{\circledast}$, utilisée dans l'instrumentation en physique nucléaire.

\section{Techno-esthétique}

10 L'esthétique du générateur (fig. 2) est liée durant sa vie active à sa capacité à remplir son rôle d'artefact dans une situation d'usage ${ }^{10}$. Il y a un rapport direct entre forme et fonction. Ce postulat est d'ailleurs relayé par deux grands penseurs de la machine moderne, Lewis Mumford ${ }^{11}$ et Gilbert Simondon, pour qui la beauté d'une machine se révèle d'autant mieux qu'elle est concrète, lorsqu'elle est toute concentrée dans une fonction, et se manifeste en action à l'endroit du monde qu'elle complète (le tracteur dans son sillon, la machine de laboratoire au sein de sa cage de Faraday, etc.). Cette esthétique peut être datée dans l'histoire de l'art avec l'avènement du Bauhaus et du design. Le dépouillement fonctionnel est en fait un des avatars $\mathrm{du} \mathrm{xx}^{\mathrm{e}}$ siècle de l'esthétique technique. Au XIX ${ }^{\mathrm{e}}$ siècle par exemple, les instruments scientifiques en laiton étaient pour la plupart vernis, ce qui les protégeait de la corrosion et des dégradations et leur conférait un aspect doré : ce caractère ornemental n'a aucun rapport avec le minimalisme formel des objets techniques $\mathrm{du} \mathrm{xx}^{\mathrm{e}}$ siècle, plus proches de l'abstraction et du futurisme. 
Figure 2

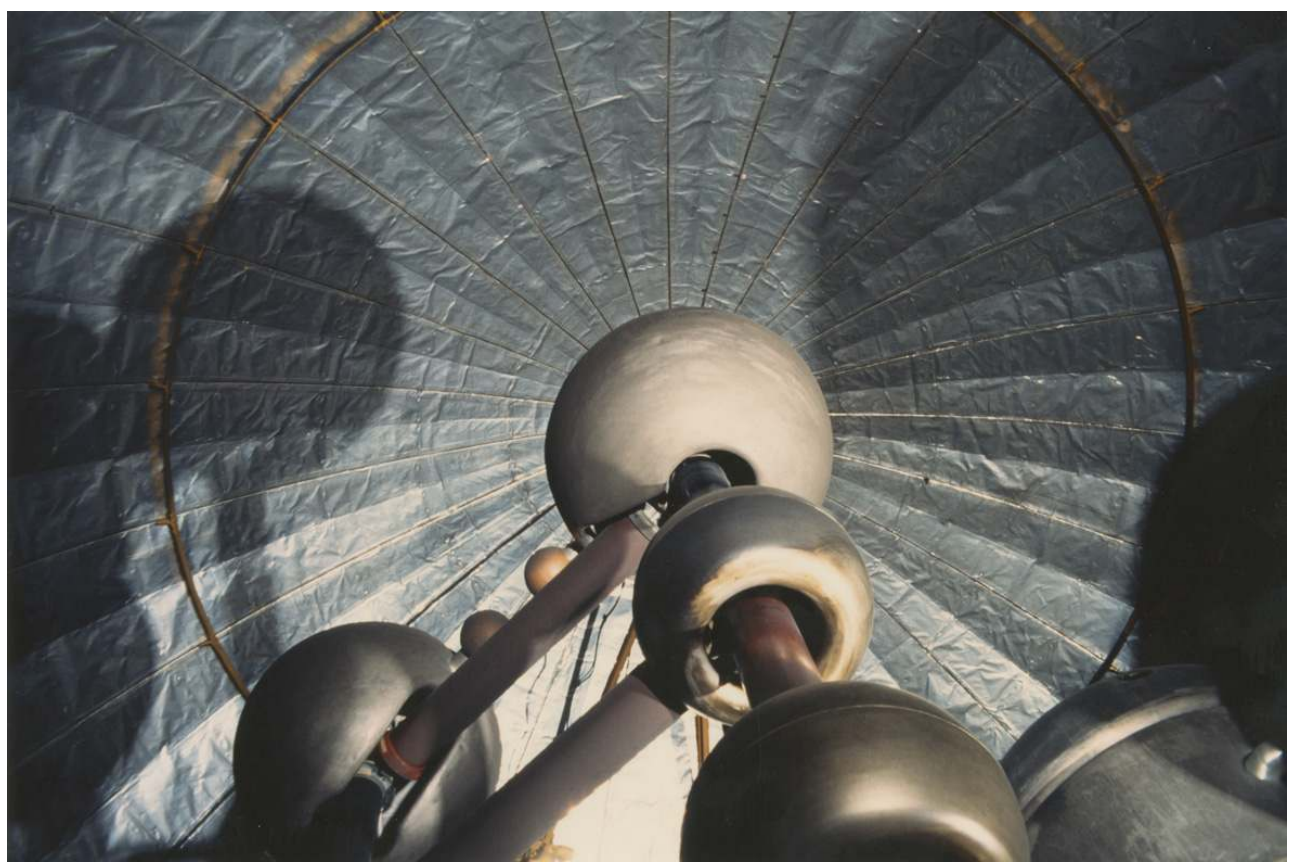

Le générateur Cockcroft-Walton dans son environnement d'usage : vue de la cage de Faraday, des pare-effluves toriques en aluminium, et des condensateurs en haefelyte.

Phot. IPNL. () IPNL, 1970.

Or, l'obsolescence de l'objet tend à faire perdre, dans la plupart des cas, les composantes essentielles du rapport forme/fonction: l'ustensilité et l'environnement d'usage. Sa valeur esthétique n'en est pas pour autant diminuée; en effet, nombre de sédiments culturels changent notre perception des objets techniques, parfois même de manière insoupçonnée. «On peut dire que le bien, dès le moment où il est extrait du lieu où il reposait, est exposé, d'une part, à une détérioration physique et, d'autre part, à une détérioration culturelle puisqu'il est transformé et se charge de multiples connotations qui ne sont pas toutes légitimes ", écrivait Umberto $\mathrm{Eco}^{12}$. Les goûts, l'histoire des styles, de l'art, nos représentations de la science et du futur, la science-fiction modifient notre regard sur les objets techniques. On peut penser à l'éclateur (cité dans l'introduction) et autres machines atomiques photographiées par Robert Doisneau dans le laboratoire des Joliot-Curie. Le générateur Cockcroft-Walton de l'IPNL véhicule un imaginaire collectif qui le rend digne de Captain Atom, du laboratoire du Dr Septimus dans La Marque Jaune ou des générateurs haute tension de la base secrète de Sbrodj dans Objectif Lune. Il a d'ailleurs été utilisé comme décor pour une série télévisée de science-fiction, Bing, diffusée sur FR3 au début des années $1990^{13}$.

\section{Méthodes d'enquête}

Quoique nous soyons contemporains de l'objet à conserver et de la société qui l'a fait naître, le générateur Cockcroft-Walton reste, à plusieurs titres, difficile d'accès tant la culture technique est souvent fantasmée et le mode d'existence de ces objets peu connu, excepté par le prisme de la science-fiction et de la vulgarisation scientifique. L'anthropologie des techniques nous apprend, notamment avec André Leroi-Gourhan 
(1982), que « toute la personnalité d'un groupe humain est enfermée dans la moindre de ses productions matérielles, comme dans toutes les œuvres de son esprit » ${ }^{14}$. Il s'agit bien de saisir la part de culture du générateur Cockcroft-Walton à la place qui était la sienne, dans son milieu, en amont de tel tube accélérateur, avec tel pupitre de commande, avec tels physiciens, dans telle cage de Faraday, tel laboratoire, tels champs disciplinaires. La définition du projet culturel et scientifique autour du générateur et son exposition dans un autre contexte ne peuvent être envisagées qu'après avoir exhumé cet environnement passé.

13 L'enquête que nous avons menée emprunte sa méthode aux sciences sociales et a pour but de pallier la perte de savoir liée à l'obsolescence de l'objet puisqu'il faut avoir, comme le rappelle Pierre Bourdieu dans le cas d'un objet de la même famille "incorporé beaucoup de théorie et aussi de routines pratiques pour être à la hauteur des demandes d'un cyclotron ${ }^{15}$ ». Le concours de l'anthropologie, de l'ethnologie, de la sociologie et de l'histoire des sciences et des techniques est donc indispensable afin de comprendre l'objet sans amenuiser une de ses qualités. L'enquête technologique propose de comprendre les éléments et pièces du générateur (organes fonctionnels, matériaux), ses usages (fonctionnement électrique, finalités scientifiques, utilisation, maintenance, obsolescence) et les dispositifs techniques dans lesquels il s'inscrit. Ces investigations invitent à multiplier et croiser les sources (en lien notamment avec le service de documentation de l'IPNL) : interviews d'anciens utilisateurs, publications scientifiques, brevets du fabricant, histoire des sciences, journaux et rapports d'activité, inscriptions sur l'objet, photographies, vidéos, plans, archives diverses, etc.

De la même manière qu'il est impossible de suivre le parcours d'un générateur CockcroftWalton sans bifurquer de la politique à l'ingénierie, de l'armement nucléaire français à la trajectoire d'une particule, on ne peut suivre le fil de la conservation du générateur sans passer d'une discipline à l'autre, disciplines que l'on tient d'ordinaire pour distinctes. Sa préservation hors du milieu, hors de l'ensemble complexe composé d'instruments, de bâtiments et d'humains, passe donc par différents savoirs : socio-culturels (des «science studies» aux mécanismes patrimoniaux), historiques, esthétiques, techniques et scientifiques. Les modalités de préservation d'un tel objet, sur lequel viennent se cristalliser autant d'aspects culturels (des physiciens atomistes à la bande dessinée), doivent résulter de cette transdisciplinarité.

\section{Biographie du générateur}

15 La littérature spécialisée s'accorde sur ce point : pour entreprendre la conservation d'un objet technique de cette ampleur, il faut avoir recomposé l'ensemble du parcours historique de l'objet, de sa conception à sa phase d'abandon. Ce travail permet notamment de définir un état de référence pour le remontage et la restauration du générateur Cockcroft-Walton. Veut-on restaurer l'accélérateur à neutrons $1,2 \mathrm{MeV}$ à 4 étages acheté par Jean Thibaud en 1953 ou bien la colonne Haute Tension surélevée d'un étage de l'accélérateur d'agrégats d'hydrogène qui donne lieu à une première mondiale en collaboration avec le CERN en 1992 ? Combien d'étages de Haute Tension doit-on remonter? Y a t-il des amalgames historiques possibles : le Cockcroft-Walton tel qu'il va être remonté peut-il être fidèle à ses différents avatars?

On peut distinguer cinq étapes historiques dans le parcours du générateur, que nous proposons de retracer (fig. 3) : la première, sa fabrication par l'entreprise Haefely en 
1952, la deuxième, au fort de la Vitriolerie, dans un contexte militaro-scientifique (1953-1963), la troisième, dans le bâtiment Haefely de l'IPNL (1963-1970), la quatrième, dans le Dôme avec l'accélérateur d'agrégats d'hydrogène (1970-1999), et enfin, la cinquième, le stockage dans le bâtiment Haefely à partir du dernier démontage (1997 à aujourd'hui).

Figure 3

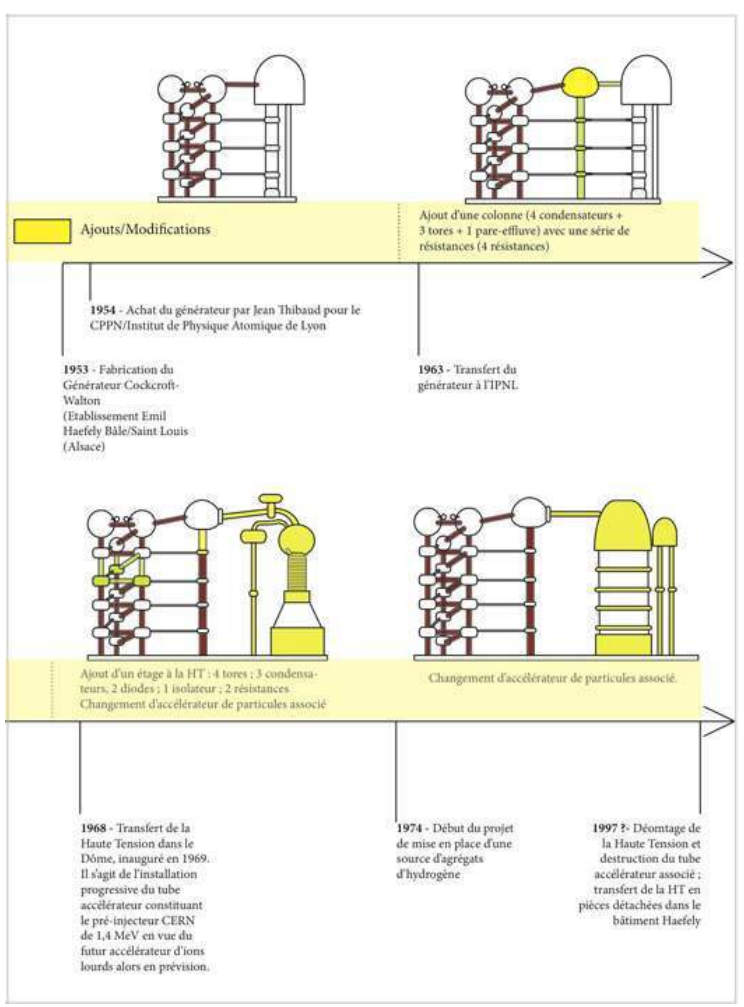

Schéma chronologique d'évolution et de modifications (en jaune) du générateur Cockcroft-Walton en quatre étapes.

Schema. Rémy Geindreau. (c) Rémy Geindreau, 2014.

$1^{\text {e }}$ étape : Jean Thibaud, qui avait obtenu en 1921 le diplôme de l'École supérieure d'électricité, soutenu une thèse de recherche fondamentale sous la direction de Maurice de Broglie (directeur du laboratoire de physique des rayons X), travaillé sur les premiers accélérateurs de particules des années 1930, fonde en 1935 l'Institut de physique atomique, rue Raulin à Lyon $^{16}$. Après la guerre, il passe commande d'un générateur Cockcroft-Walton qui sera construit en grande partie par les établissements d'ingénierie électrique Emil Haefely à Bâle (Suisse), seuls les condensateurs ont été fabriqués en 1953 par la filiale française de l'entreprise à Saint-Louis, en Alsace. Selon M. Métraux, directeur de Haefely l'année de l'achat du générateur, sa fabrication constitue une première technique: "c'est la première fois qu'un redresseur d'un potentiel de $1200000 \mathrm{~V}$ est réalisé à l'aide d'éléments secs $»^{17}$.

$2^{\text {e }}$ étape : En 1951, un accord entre l'Armée et l'Institut de physique atomique (par l'intermédiaire de J. Thibaud) permet l'installation au fort de la Vitriolerie d'un établissement de recherche ayant pour mission de former des spécialistes militaires atomistes, nommé Centre de perfectionnement en physique nucléaire (CPPN). Le générateur Cockcroft-Walton et le tube accélérateur associé (fig. 4) y ont été mis en 
service en $1953^{18}$. Ce centre est remplacé en 1955 par le Centre de perfectionnement atomique des armes spéciales (CPAAS).

Figure 4

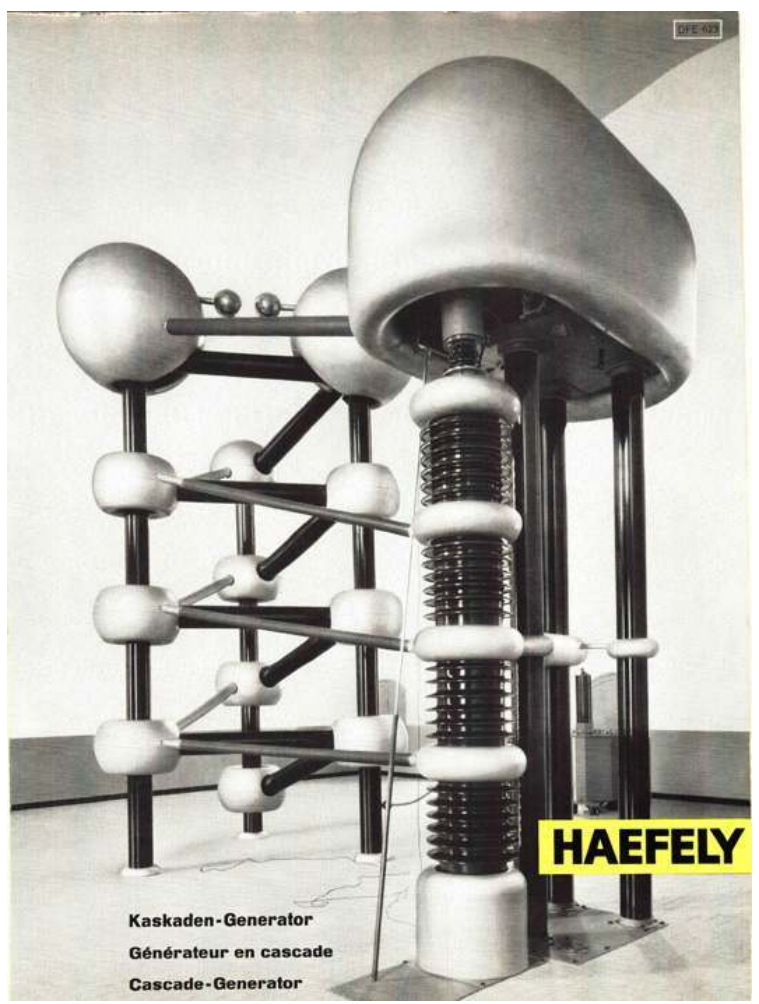

BROCHURE DE PUBLICITÉ DU FABRICANT HAEFELY AVEC LE gÉNÉRATEUR COCKCROFT-WALTON DE L'IPNL ALORS AU FORT DE LA VITRIOLERIE (CENTRE DE PERFECTIONNEMENT EN PHYSIQUE NUCLÉAIRE).

Phot. Haefely. (C) Haefely, 2014.

$193^{\mathrm{e}}$ étape : Jean Thibaud fonde en 1961 le premier centre de physique nucléaire en province (IPNL) sur le campus de la Doua à Lyon (fig. 5). Le générateur y est transféré en 1962 par le fabricant et quelques techniciens de l'Institut. Il est placé dans le bâtiment " Haefely », construit en fonction de ses dimensions pour l'accueillir.

Cependant J. Thibaud ne devait voir que l'installation du Haefely et ne connaitre que les premiers travaux. [...] Après une période de formation (de l'accélérateur et du personnel...) puis d'étalonnage, pendant laquelle Jean Thibaud montra encore son sens de l'expérience et des dispositifs simples et efficaces, l'accélérateur fut surtout utilisé comme générateur de neutrons ${ }^{19}$. 


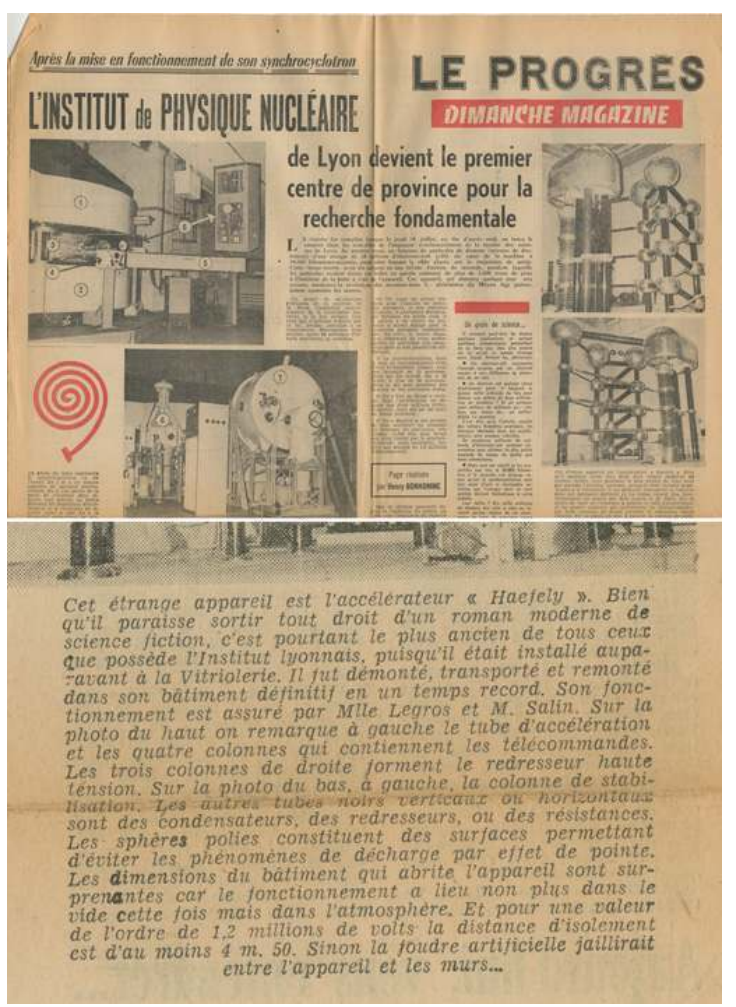

Article paru dans Le Progrès - dimanche magazine, lors de l'inauguration de l'Institut de physique nucléaire de Lyon sur le campus de la Doua. On y voit le générateur Cockcroft-Walton avec une nouvelle colonne de stabilisation. Agrandissement sur la légende « Cet étrange appareil... ».

Art. Henry Bonhomme. (c) Le Progrès, 1963.

$4^{\mathrm{e}}$ étape : À partir de son transfert dans le Dôme ${ }^{20}$ (un hall hémisphérique construit en 1968 pour accueillir le générateur et ses dispositifs de contrôle), l'objet change en partie de fonction. Un cinquième étage est ajouté, selon la proposition de M. Salin, afin d'améliorer la stabilité de la tension en sortie (il y en avait quatre jusqu'ici ; chaque étage de condensateurs et de pare-effluves fait 1,20 m de hauteur). Cet ajout va obliger les physiciens et techniciens à réduire la tension du générateur de $1,2 \mathrm{MV}$ à $630 \mathrm{kV}$. C'est dans ce cadre qu'ont lieu les essais de la colonne accélératrice mise au point par le CERN et l'IPNL (pré-injecteur du projet linéaire). Selon le Courrier du CERN,

La collaboration avec Lyon portait principalement sur le tube accélérateur, l'IPNL fournissant une partie des moyens d'essais dont un imposant hall servant de cage de Faraday et un générateur $1,5 \mathrm{MeV}$, et le CERN apportant le dispositif expérimental et son expérience dans la construction et l'exploitation des tubes accélérateurs ${ }^{21}$.

$5^{\mathrm{e}}$ étape : Enfin, les faibles performances du générateur l'ont condamné à l'obsolescence. Selon J. Martin « une étude est entreprise pour orienter l'accélérateur dans une autre direction et créer un nouveau bâtiment annexe mais ces modifications ne sont pas acceptées, c'est la mort de la machine ! Son démontage est entrepris en $1997 »^{22}$. Le tube accélérateur du Dôme sera détruit, le générateur démonté, étiqueté (ce qui indique l'existence d'un plan de montage dont nous n'avons pas retrouvé la trace à ce jour) et transféré dans le bâtiment Haefely (fig. 6). L'objet est en partie conservé dans des caisses en bois réalisées lors du dernier démontage sous la direction de M. Martin. C'est durant 
cette phase d'abandon que les principales altérations vont être constatées. Succinctement, un constat d'état de l'objet en 2013 fait apparaître: une couche importante de poussière (particules fines, matériaux divers...), des dépôts graisseux sur l'ensemble des pièces, des rayures, un ternissement et des déformations des sphères en aluminium, l'effacement complet des étiquettes placées au démontage, des empreintes de doigts, des traces brunes liées à l'application d'un produit de nettoyage (probablement utilisé pour polir les sphères), la corrosion de certains éléments en acier (structure des pare-effluves), une patine des alliages cuivreux et, enfin, quelques fuites d'huile contenu dans les condensateurs.

Figure 6

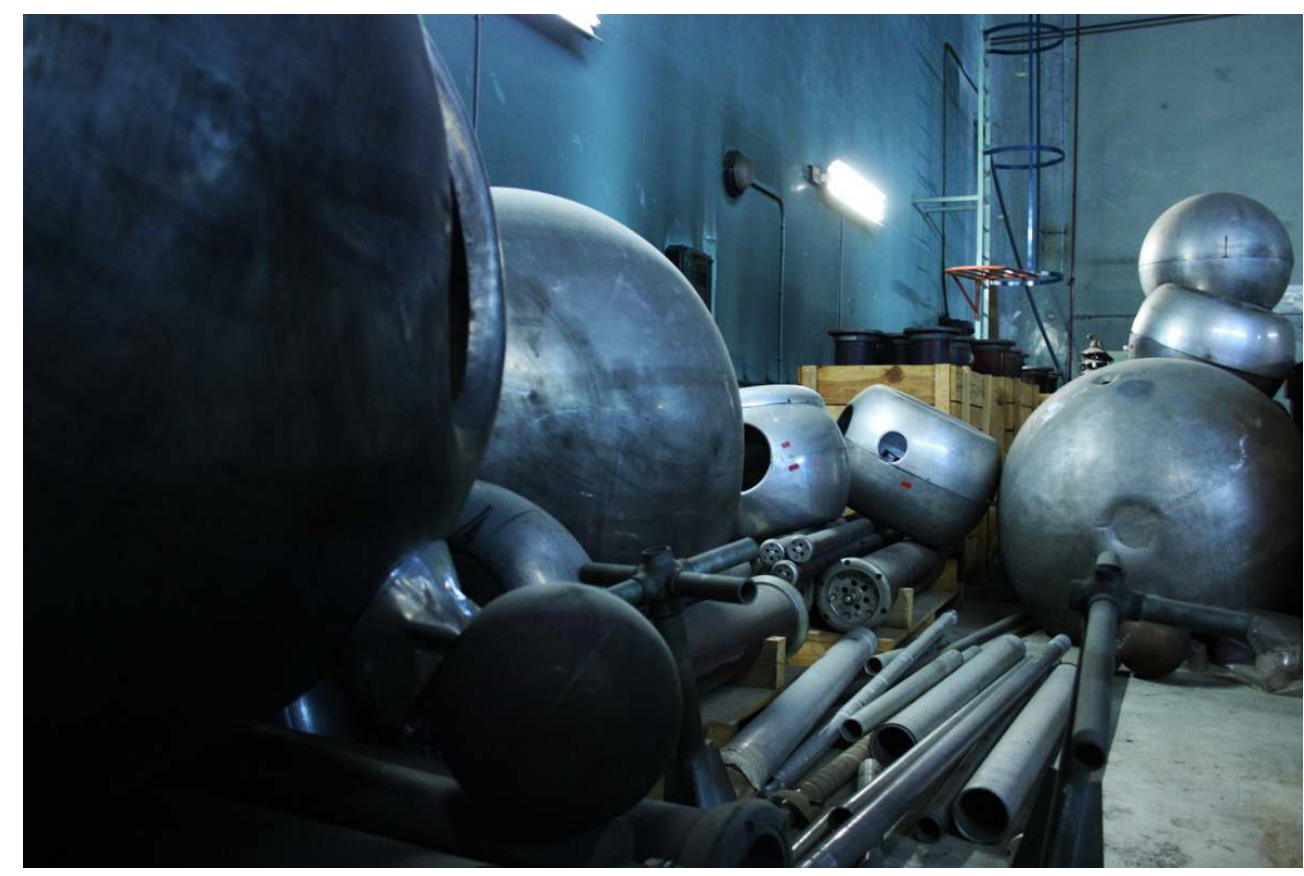

Le générateur Cockcroft-Walton tel qu'il est aujourd'hui, en pièces détachées (pare-effluves, condensateurs, diodes, résistances) dans un laboratoire en activité.

Phot. Séverine Derolez. ( IPNL, 2013.

\section{Un environnement disparu : machines, hommes, bâtiments}

Le générateur tel qu'il est aujourd'hui, fragmenté et dissocié, ne représente qu'une infime portion de l'ensemble initial. Le réseau dans lequel il s'inscrit fait lui-même partie d'autres réseaux (scientifiques, universitaires, militaires et désormais culturel). L'instrument appartient à un " champ d'activité technique ${ }^{23}$ " vaste et hétérogène à la fois composé d'humains et de non-humains ${ }^{24}$. Ce milieu associé est la condition sine qua non d'existence de l'objet. Il était composé d'un redresseur/multiplicateur à très haute tension (le générateur Cockcroft-Walton), d'une source de protons et d'un tube d'accélération avec les cibles (fig. 7), des dispositifs accessoires (cannes, ponts roulants, signalétiques, éclateurs, transformateurs, éléments de rechange, bouteilles de deutérium, plaques photographiques, etc.). Les "manips" étaient contrôlées depuis un pupitre central (fig. 8) qui comportait tous les instruments de mesure et les organes de commande nécessaires. Le ferraillage des tubes accélérateurs associés au générateur en 
1997, de l'accélérateur 1,2 MeV de Haefely à l'accélérateur d'agrégats d'hydrogène du Dôme, constitue le plus grand défi du point de vue de la présentation de l'objet.

Figure 7

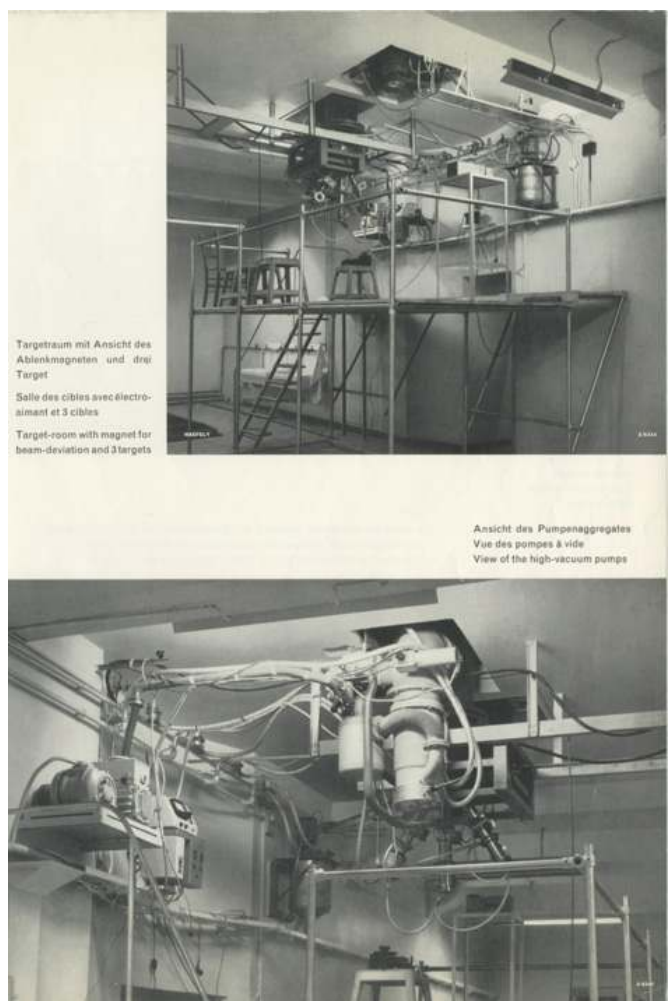

Salles des cibles avec électroaimants placés sous le générateur Cockcroft-Walton au fort de la Vitriolerie (Centre de perfectionnement en physique nucléaire) de 1953 à 1963. Brochure de publicité du fabricant Haefely.

Phot. Haefely. (c) IPNL/Haefely, 2014. 
Figure 8

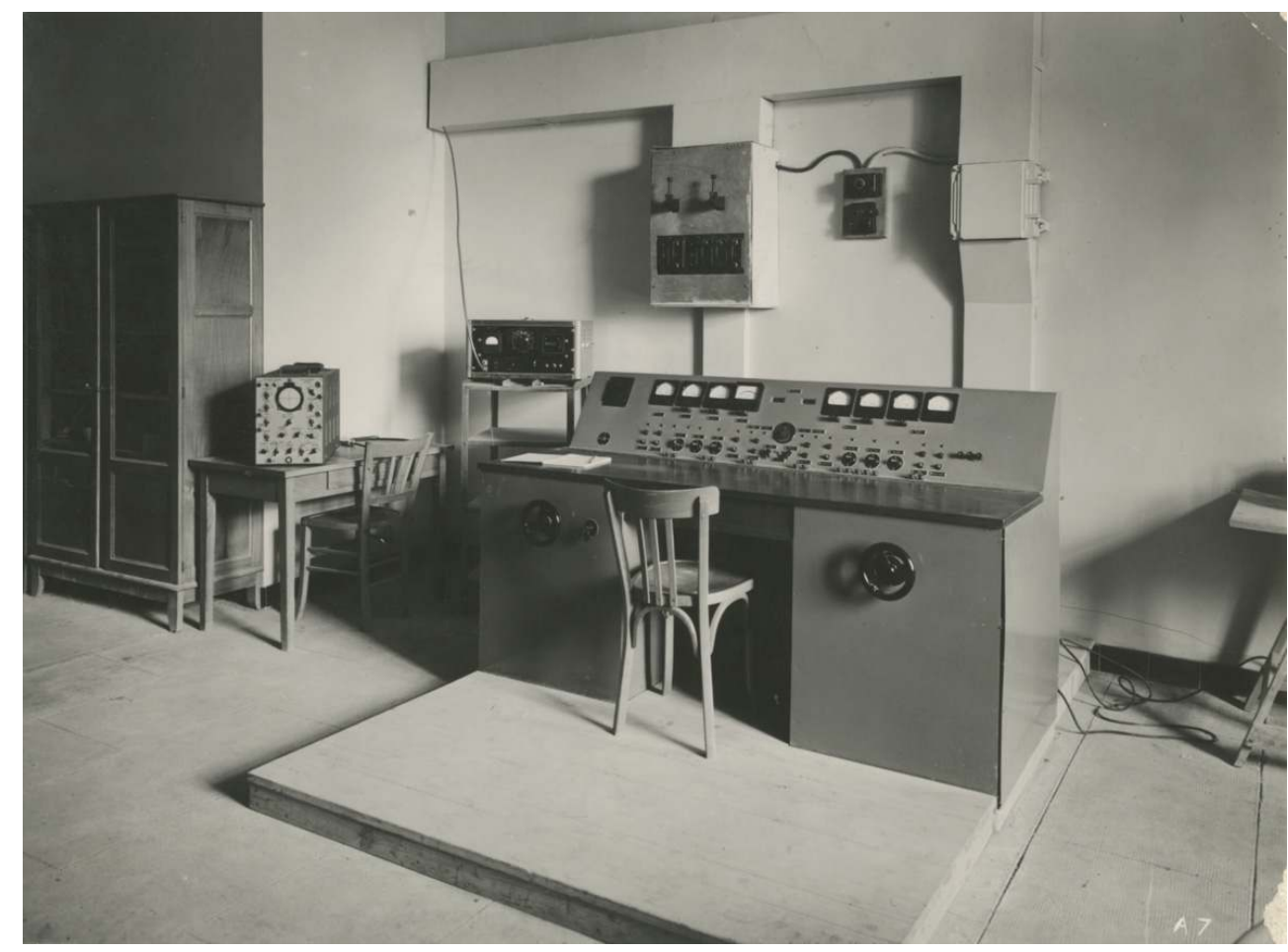

Le pupitre de commande du générateur Cockcroft-Walton au fort de la Vitriolerie (Centre de perfectionnement en physique nucléaire) de 1953 à 1963 utilisé notamment dans la brochure de publicité du fabricant Haefely.

Phot. IPNL. () IPNL, 1954.

De nombreux physiciens, ingénieurs, techniciens, étudiants se sont succédé pour faire fonctionner le générateur Cockcroft-Walton, de son achat militaro-scientifique aux expériences en physique expérimentale dans les locaux de l'IPNL. On peut citer de manière non-exhaustive: Mlle G. Legros, M. Salin, J. Depraz, G. Burnot, M. Dufay, M. Lafoucriere, M. Martin, M. Lopez, M. Filiol, M. Farizon... Les collectifs diffèrent suivant les programmes de recherche et «le temps de faisceau » est découpé entre les différentes équipes. Les gestes, les manipulations, les règles, les modes opératoires nous sont pour la plupart méconnus, ne subsistent que les publications scientifiques, les journaux d'activités et les témoignages des anciens utilisateurs (avec les difficultés et contradictions liées à la transcription d'une pratique de laboratoire en mots).

Trois ensembles architecturaux, comme nous l'avons vu, ont accueilli le générateur : le fort de la Vitriolerie (a) de 1953 à 1962 ; le bâtiment «Haefely " (b) de 1962 à 1969; et enfin, le Dôme (c) de 1968 à 1997 :

a) Les salles du fort de la Vitriolerie (quartier général Frère à Lyon) construit au XIX siècle ont été adaptées à l'objet: destruction des sols, reconstruction de la voûte, etc. Selon des sources orales, les arcs électriques étaient nombreux du fait de l'humidité élevée à proximité du Rhône.

b) Le bâtiment « Haefely » est construit sur la plaine de la Doua en 1961 en même temps que les bâtiments des autres accélérateurs de particules «Synchro-cyclotron » et «Van de Graaff» (fig. 9). Les architectes d'opération sont Alain Chastel et Abel Cholat sous la houlette de Jacques Perrin-Fayolle. Les murs du bâtiment « Haefely » sont composés d'une 
gangue de béton de plus de 1,50 $\mathrm{m}$ placée à plus de $4 \mathrm{~m}$ de distance de l'objet (ce qui explique ses grandes dimensions). L'extérieur est composé d'éléments de céramique noirs en forme de pointe ; c'est pourquoi Le progrès de Lyon du 18 septembre 1961 qualifie les bâtiments de "gros diamants noirs ».

Figure 9

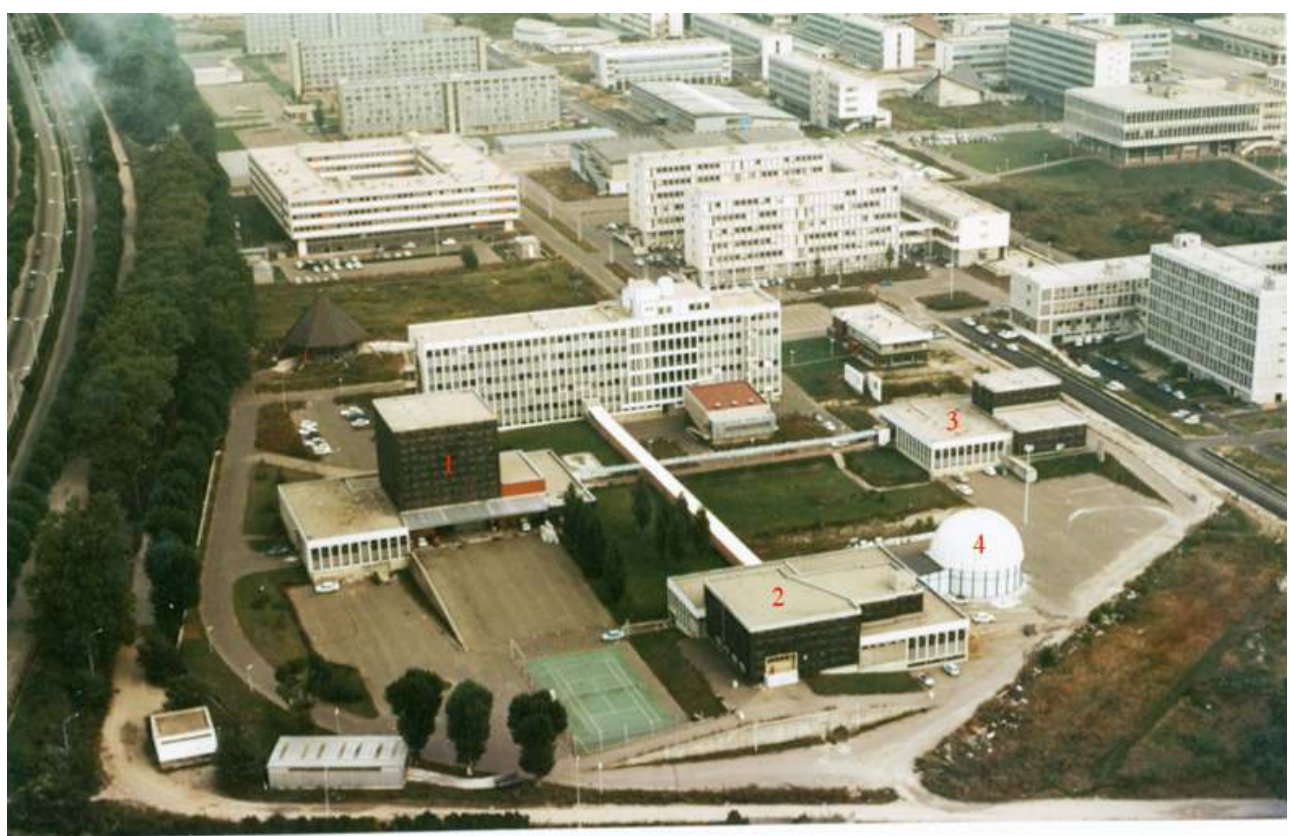

\section{UNIVERSITE DE LYON _ _ INSTITUT DE PHYSIQUE NUCLEAIRE}

Vue aérienne de l'Institut de physique nucléaire de Lyon sur le campus de la Doua. Légendée par nos soins (2015) : le bâtiment « Haefely » (1), le bâtiment « synchro-cyclotron » (2), le bâtiment «Van de Graaff » (3) et le « Dôme » (4) qui ne sera construit qu'en 1969.

Phot. IPNL. () IPNL, 1969.

c) Le «Dôme » (fig. 10, fig. 11) a été conçu par Jean Roux, ingénieur au laboratoire, et Jean Martin, il est inauguré le 27 juin 1969 par A. Sarazin, directeur de l'IPNL. Il est élaboré en fonction de sa rapidité d'exécution, de son faible poids et de son coût réduit. Le toit de cette gigantesque cage de Faraday est en mousse de polyuréthane et polyester dont la face intérieure est revêtue d'aluminium de $0,1 \mathrm{~mm}$ d'épaisseur. Il s'agit d'une structure adaptée à la hauteur des colonnes du générateur (calcul des équipotentielles : respect d'une distance de $4 \mathrm{~m}$ entre le générateur et la paroi du dôme), elle forme une enceinte démontable empêchant l'appareillage de polluer son milieu (nuisances électriques et électromagnétiques). 
Figure 10

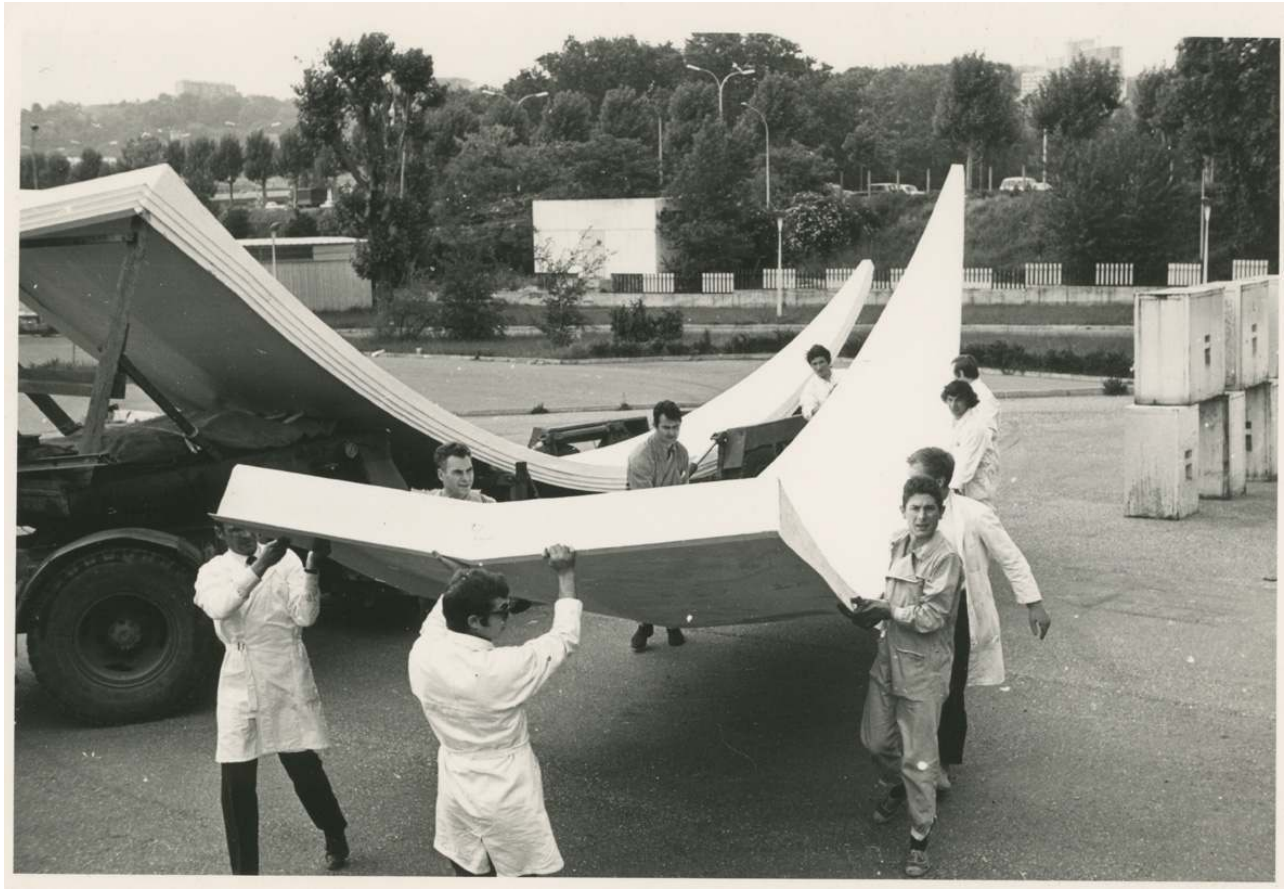

Déplacement de la structure en polyester du « dôme ». Construction du hall hémisphérique adapté aux dimensions du générateur Cockcroft-Walton.

Phot. Auteur inconnu. (C) IPNL, 1969.

\section{Figure 11}

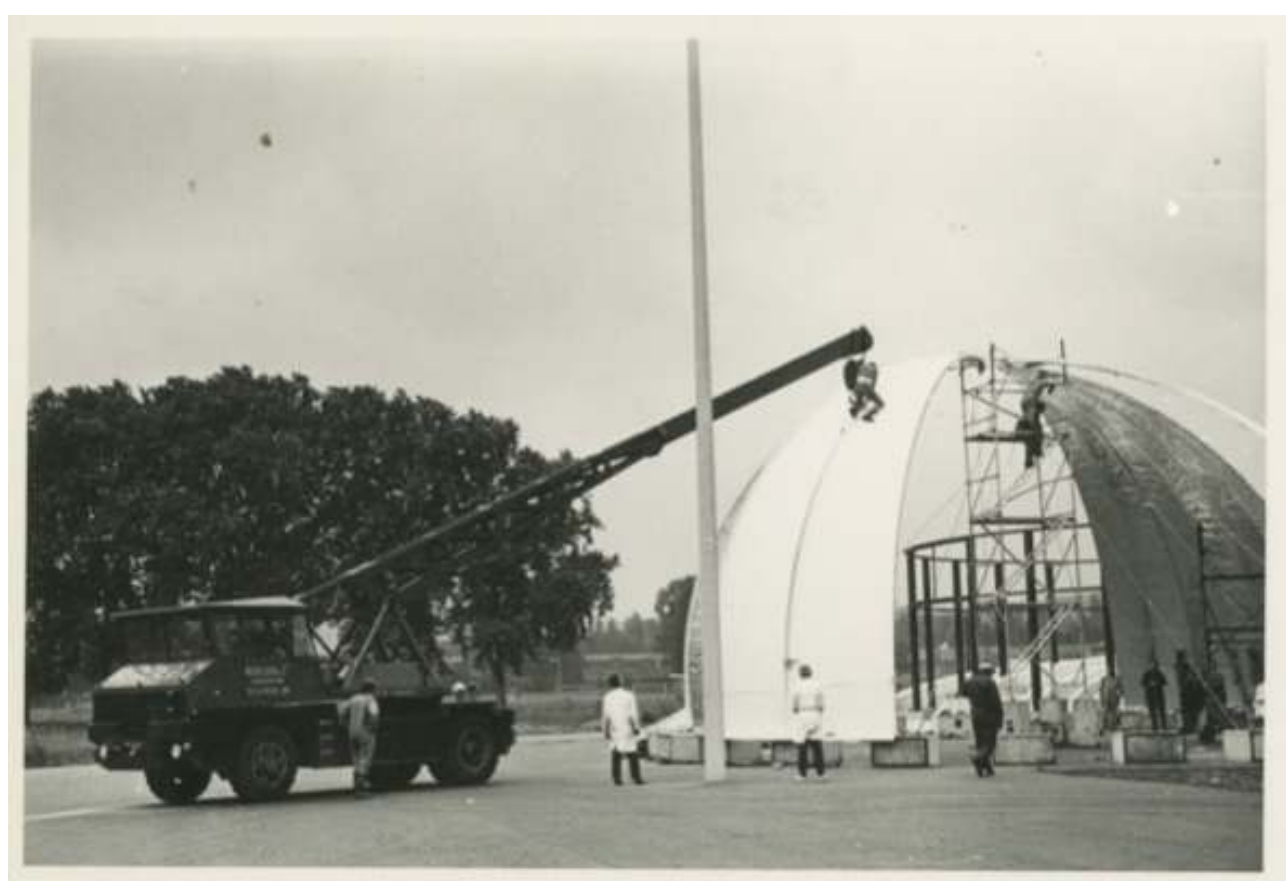

Construction du hall hémisphérique aux dimensions du générateur Cockcroft-Walton.

Phot. Auteur inconnu. (c) IPNL, 1969. 


\section{Les perspectives}

28 ceux de Cambridge, ceux utilisés pour le Manhattan project à Los Alamos, ceux utilisés au CERN, etc.), par son témoignage historique (la technicisation de la science, la physique nucléaire en France, Jean Thibaud, évolution de l'IPNL...) et ses connotations esthétiques avait, dès son démontage, intéressé les musées des techniques. Il cristallise aujourd'hui la mémoire des recherches, des travaux, mais également la mémoire des physiciens, au-delà même de son rapport étroit avec l'histoire des sciences et des techniques. Le fait que l'IPNL l'ait préservé, bien qu'il occupe une place considérable dans un laboratoire en activité, est assez significatif sur ce point. L'objectif aujourd'hui est d'exposer l'objet en tant que support d'enseignement et de diffusion culturelle à destination d'un public large ; en faire, en quelque sorte, un emblème du département de physique de l'université sur le campus de LyonTech-la Doua.

L'inscription de l'objet au titre des monuments historiques qui a été évoquée lors de l'une des premières réunions du " groupe patrimoine physique ", n'est malheureusement pas à l'ordre du jour. Les personnes chargées de la sauvegarde de l'appareil sont toutes bénévoles et en poste (enseignants chercheurs, chercheurs retraités...) et ne bénéficient pas de décharge de temps pour cette mission. De plus, l'intérêt de l'université pour son patrimoine est récent et ne permet pas aujourd'hui de mobiliser des moyens humains pour monter le dossier de classement (s'approprier la réglementation et la législation) et d'en assumer les conséquences. Les travaux en cours autour de cet appareil (cahier des charges à la restauration / modélisation 3D / thèse d'histoire des sciences / thèse de didactique des sciences) ${ }^{25}$ pourraient constituer une documentation de base pour un éventuel classement de l'objet dans les années à venir.

Les solutions de préservation du générateur sont tributaires du projet culturel associé. Sa définition par l'ensemble des acteurs est indispensable. Il est conditionné par l'université Claude Bernard Lyon 1, par les moyens humains, matériels et financiers mis à disposition, et découle des résultats des enquêtes historiques et ethno-technologiques. Une étude comparative des choix muséographiques des institutions qui présentent des générateurs Cockcroft-Walton nous convainc de la grande variété des solutions qui peuvent être proposées. Certains font partie des expositions permanentes des musées scientifiques comme au Science Museum de Londres, au National Scotland Museums d'Edinburgh, ou encore à Madrid dans le Museo Nacional de Ciencia y Tecnologia Muncyt. L'un d'entre eux a même trouvé sa place au sein d'un musée de "Sciences Arts et Sociétés» dans l'exposition Sociétés : le théâtre des hommes du Musée des confluences (Lyon). La médiation, développée autour de l'accélérateur Philips des années 1950, fait d'ailleurs l'objet d'étude d'une thèse en cours à l'Université de Lyon. D'autres ont été remisés en extérieur comme au CERN ou l'IPHC de Strasbourg, d'autres s'inscrivent dans le cadre de musées universitaires comme au Cavendish Laboratory avec un accélérateur fabriqué par Haefely ou au museo della tecnica elettrica de Pavie (Italie). L'exemple de la Boule de Rangueil du Centre d'élaboration de matériaux et d'études structurales (CEMES-CNRS) à Toulouse avec un microscope électronique à très haute tension (1958) nous semble tout à fait pertinent (du point de vue de la conservation) par rapport au projet que nous menons. L'objet, comprenant une haute tension de type Cockcroft-Walton, est toujours dans sa cage de Faraday (la boule de $25 \mathrm{~m}$ de diamètre) inaugurée en 1959 par le général de Gaulle. Ce 
microscope, aujourd'hui hors service, s'il ne fait aujourd'hui l'objet d'aucun projet muséographique, est présenté au public lors des journées d'ouvertures exceptionnelles (journées du patrimoine, fêtes de la science...) ou sur réservation.

\section{Dilemmes de la conservation-restauration}

31 L'étude préalable à la restauration du générateur Cockcroft-Walton (constat d'état, diagnostic et pronostic sur l'évolution des altérations, analyses scientifiques des matériaux, étude et hiérarchisation des valeurs accordées à l'objet, propositions de traitements et de maintenance, protocole de remontage, mesures de conservation préventive...) a pour dessein, dans un premier temps, de clarifier les conditions et moyens de conservation matérielle de l'objet. Pour ce type de projet, le travail du conservateurrestaurateur peut s'avérer utile en amont, pour rédiger un cahier des charges à destination d'autres spécialistes (ici les physiciens et techniciens du laboratoire). D'autant plus que l'efficacité de l'objet dans le champ patrimonial dépendra, par la suite, de notre capacité à faire collaborer différents acteurs pour sa restauration. Il ne faut pas opposer les savoirs de ceux qui ont maintenu et réparé, aux savoirs de ceux qui vont préserver et restaurer ${ }^{26}$ : force est de constater qu'ils se confondent parfois bien que les compétences et les objectifs diffèrent. Il y a une adéquation entre l'ingénieur électrotechnicien qui va tenter de réduire l'ondulation résiduelle de la tension du générateur et le restaurateur qui va déterminer les paramètres de remontage et de conservation du même objet.

Si la rédaction d'un cahier des charges suppose, entre autres, des connaissances dans les traitements d'inhibition des alliages d'aluminium et dans la protection de l'haefelyte des condensateurs, nombre de questions dépassent les seuls problèmes relatifs aux procédés de traitement, bien qu'elles les alimentent. Face aux problèmes évoqués liés à l'environnement de présentation en atmosphère mal ou non contrôlé, à la diversité des matériaux modernes (dont le vieillissement est encore mal connu), à l'échelle et aux usages passés de l'objet, le monopole de la conservation-restauration ne va pas de soi. Non seulement parce que la réalisation des traitements est souvent le fait d'entreprises spécialisées (ce n'est pas le restaurateur qui réalise seul l'ensemble des opérations) mais aussi parce que le praticien peut souffrir d'un déficit théorique et méthodologique face à l'agrandissement du champ patrimonial. La discipline de la conservation-restauration telle qu'elle s'est construite collectivement émane, en partie, d'une conception de l'objet en tant qu'œuvre des beaux-arts « classiques ». Conception qui s'accorde mal, ou sinon à renfort de contorsions, avec la restauration de la partie haute tension d'un accélérateur de particules.

La conservation-restauration des objets techniques, qui tend à se constituer en spécialité à part entière, oscille historiquement entre deux positions; ou bien une remise en fonction des machines qui s'apparente à une réparation, ou bien une stabilisation de type historique de l'objet (préservation dé-fonctionnalisée) ce qui est souvent le cas dans les musées ${ }^{27}$. Cet état de fait en dit long sur les spécificités de ces artefacts pour lesquels il existe non pas un type de restauration normée mais des restaurations plurielles. La première de ces approches est vite mise en échec face à un objet issu de la "big science » ${ }^{28}$. En effet, remettre en fonction le générateur Cockcroft-Walton relève de l'utopie sauf si l'on fait abstraction des nombreuses contraintes techniques (obsolescence de certains composants comme les redresseurs), de sécurité, et si l'on considère que les ajouts de pièces neuves ne sont pas actes de falsification. De surcroît, le courant électrique (aussi 
visuelle que puisse être la métaphore langagière du «courant») qui traverse les condensateurs et diodes du générateur, est invisible. Que pourrait-on voir de plus, nonobstant les phénomènes d'arcs électriques dangereux ? D'ailleurs, aucun physicien n'a pu voir le générateur sous tension, son accès étant interdit en raison des risques de radiation.

Même s'ils ne fonctionnent plus et même s'ils sont détachés de leur lieu d'utilisation, les objets techniques patrimoniaux (comme la plupart des objets conservés dans les musées qui ont une utilisation qui diffère de celles pour lesquelles ils ont été conçus) gardent un usage au mieux symbolique ou au moins documentaire. Le fonctionnement patrimonial empêche en partie le fonctionnement technique de l'objet ${ }^{29}$. Ainsi, le rôle du conservateur-restaurateur revient, ici, à établir un pont entre ces deux modes de fonctionnement.

Cet objet identique et inédit à la fois est vidé en son centre, par où toute parole peut se proférer désormais, fluer, comme si tout était dès lors possible, comme si la citadelle était à prendre. (...) Les mots vont le réinstaller dans la loi de la gravité ; les étiquettes vont solidement l'arrimer dans un sol neuf ${ }^{30}$.

Cette transplantation suppose de ne réduire l'objet technique ni à un fonctionnement (qui ferait abstraction des pannes, de la mise hors tension, des réparations, etc.) ni à une histoire (le dilemme réparer/restaurer sus-cité). C'est pourquoi, dans ce cas, la démarche de conservation-restauration doit être pensée non plus comme une spécialisation technicienne cantonnée à la stricte matérialité d'un bien culturel, mais plutôt comme une ingénierie spécifique au mode patrimonial qui découle directement des enquêtes menées sur et autour de l'objet. Les traitements doivent maintenir, dans le moindre acte sur la matière du bien (remontage, vidange de l'huile, nettoyage, etc.), son identité culturelle, historique, esthétique et technique en introduisant une symétrie entre les usages passés de l'objet et ses usages futurs. Comme il n'existe pas de restauration sans perte ni de constitution de patrimoine sans sacrifice, la résolution des conflits entre ces différents modes revient à un parti-pris technologique qui résulte d'un compromis entre les différents acteurs. En ce sens, la méthode au cas par cas est plus proche de celle développée pour la restauration des objets ethnographiques, des instruments de musique ou des œuvres d'art contemporain (notamment les œuvres à composantes technologiques). Comment, par exemple, donner à voir la fonction du générateur Cockcroft-Walton sinon par le maintien d'un potentiel utilitaire : en remontant l'objet, en rendant visibles les matériaux (sans masquer les surfaces avec des revêtements), en préservant les traces d'usage qui témoignent de l'utilisation et donc de l'historicité de l'objet? Comment transmettre le schème de l'objet technique sans prêter une attention accrue à son environnement de présentation? Ou encore, comment rendre compte du tissu de relations nouées autour de cet objet en usage social, sans en recenser, en connaître, voire en mobiliser les usagers?

Nous proposons, pour que l'objet témoigne de ses usages passés (vocation patrimoniale), de le remonter (protocole de remontage de l'ensemble des pièces détachées) tel qu'il était dans le Dôme à la fin des années 1990 avant son démontage, avec 5 étages de tension. Dans notre cas, les traitements curatifs et préventifs doivent en priorité enrayer les dégradations évolutives (corrosion, salissures, fuites...), préserver les traces d'usage (impacts d'arcs électriques, inscriptions, etc.) et l'aspect fonctionnel des éléments (la techno-esthétique expliquée plus haut qui permet, en outre, au futur public de compléter par l'imaginaire les usages de l'objet), garants de l'identité de l'objet. Ces actes, 
essentiellement de nettoyage, de stabilisation et de remontage, ne seront durables et véritablement efficaces que lorsque les conditions de conservation préventive seront stables et adaptées.

\section{Un nouveau lieu pour le générateur}

37 Le lieu d'exposition du générateur (non défini à ce jour) est décisif puisqu'il influe sur les risques (humidité, ensoleillement, vandalisme...) auxquels va être confronté l'objet et donc sur les choix de traitement. Faute de place, le générateur pourrait être remisé en extérieur. C'est, par exemple, l'option qui a été choisie par le CERN pour l'exposition d'un générateur Cockcroft-Walton historique de type Philips ou l'IPHC (Institut pluridisciplinaire Hubert Curien) de Strasbourg avec un générateur de $1 \mathrm{MeV}$ construit par les allemands en 1943 à l'Hôpital civil, aujourd'hui exposé en extérieur sur le campus de Cronembourg. Dans les deux cas, l'opération a été réalisée par des équipes internes des laboratoires. Les moyens considérables devant être déployés pour une transplantation en extérieur d'un tel objet (mise à distance, soclage, entretien...), ainsi que les modifications importantes de la matière du bien (recouvrement complet des matériaux avec des couches protectrices) couplés à l'extraction plus prononcée de l'objet de son contexte, nous invitent à émettre de nombreuses réserves. Dans notre cas, une telle présentation, hors laboratoire, sur la pelouse du campus, reviendrait à tronquer par deux fois l'identité du générateur rendu indiscernable d'une sculpture futuriste. En outre, les matériaux ne sont pas adaptés à une atmosphère non contrôlée (intempéries, pollution, risque d'infiltration...). L'altération, notamment de l'haefelyte serait alors irréversible. Un agent hydrophobe visant à limiter la présence d'eau qui pourrait s'infiltrer dans les microfissures, serait alors nécessaire. Un témoignage du fabricant nous indique d'ailleurs une vulnérabilité accrue du matériau liée au gonflement du papier en milieu humide. Des tests et analyses des moyens de protection devront être menés. Quoi qu'il en soit, un protocole de surveillance de l'objet, s'il est remisé en extérieur, devra être établi et respecté.

38 Les possibilités de présentation de l'objet en intérieur permettent - même si à ce jour, aucun endroit assez grand n'a été trouvé pour pouvoir accueillir le générateur - de ne pas limiter l'objet à une esthétique ou à une simple anecdote formelle. La possibilité de remonter le générateur avec moins d'étages de tension pour qu'un bâtiment existant puisse l'accueillir est étudiée, comme cela a pu être fait pour les générateurs de type Cockcroft-Walton du National Scotland Museum et du musée des Confluences.

D'autres hypothèses sont également examinées. Par exemple, l'ensemble architectural historique (le Dôme) qui était indispensable au fonctionnement de l'objet serait idéal pour l'exposer aujourd'hui tant du point de vue de la conservation préventive que du propos muséographique. A l'heure actuelle le Dôme est encore utilisé par les laboratoires du campus lyonnais. L'investissement des lieux est impossible. La possibilité de construction d'un hall hémisphérique en tant que salle de montage et de présentation, pourrait remédier à ce problème. L'exploitation du cahier des charges de l'époque de la construction du bâtiment, que nous avons retrouvé (fig. 12), permettrait de reconstituer un abri proche de l'environnement d'usage passé de l'objet, un contrôle des conditions de conservation (humidité relative, température, vol/vandalisme, polluants, lumière, etc.) et une adaptation à l'accueil du futur public. 


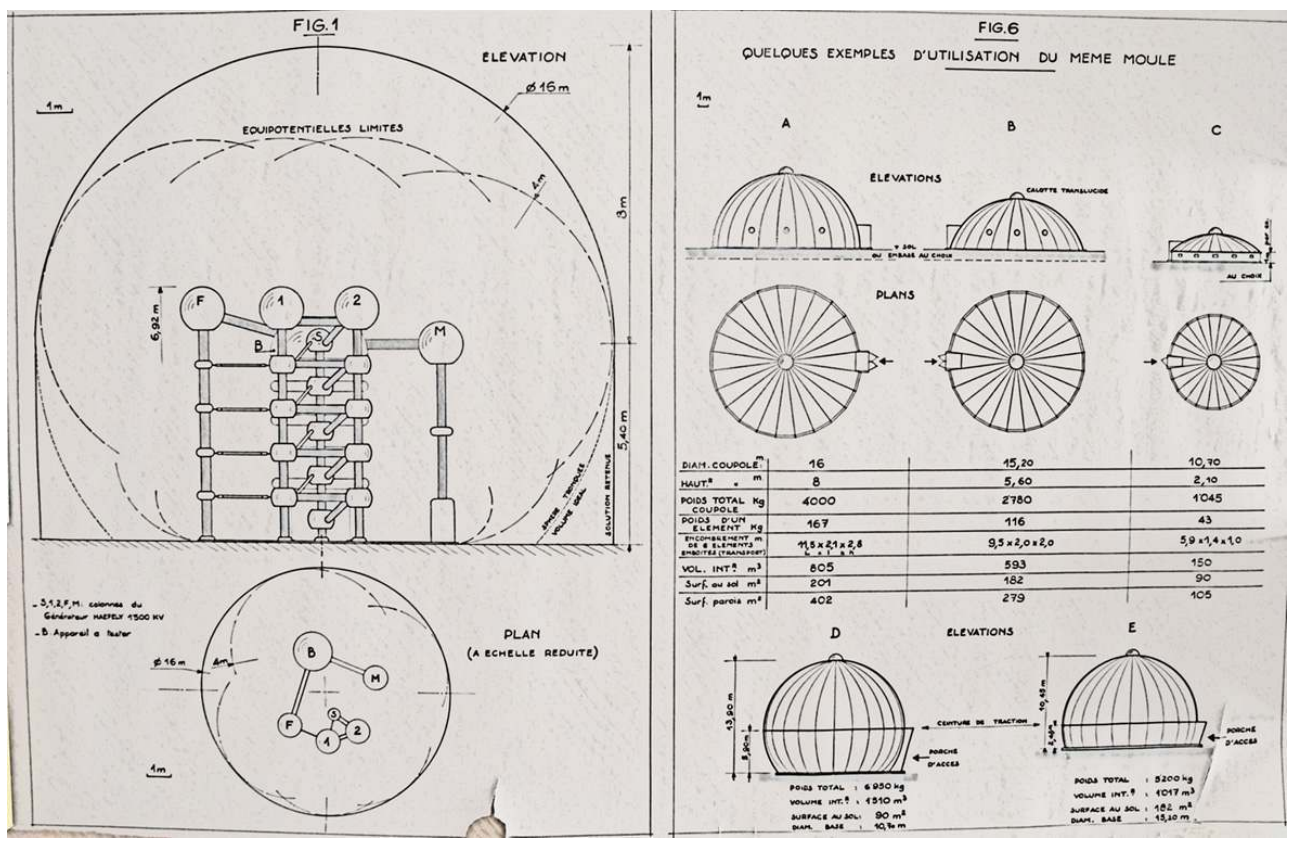

Plans du hall hémisphérique (dit « dôme ») construit aux dimensions du générateur Cockcroft-Walton. Plan. ROUX, Jean, MARTIN, Jean. (C) IPNL, 1969.

\section{Contextualisation}

Comment redonner à voir l'ensemble du réseau socio-technique disparu? Afin d'exposer un ensemble cohérent, les éléments annexes à la colonne Haute Tension pourront être présentés aux côtés du générateur : les éclateurs, les transformateurs, la signalétique, etc. Par ailleurs, quoique les traitements proposés soient soucieux par exemple de ne pas masquer les surfaces, l'objet va perdre une part de son potentiel du fait de sa décontextualisation et du fait que l'accélérateur ne peut plus être complet. C'est pourquoi l'effort doit être porté sur la mise en contexte pour comprendre les usages de l'objet, son interdépendance avec un milieu disparu et fournir des outils didactiques ${ }^{31}$. La documentation relevée durant nos enquêtes simultanées, qui a été compilée et archivée, pourra servir de support à cette médiation. Le département de physique de l'IPNL soutient, en parallèle du remontage réel de l'objet, trois projets de modélisation, qui ont déjà été entamés lors de l'élaboration du protocole de remontage de l'objet (fig. 13) : 1) une représentation informatique 3D du générateur Cockcroft-Walton complet; 2) la réalisation d'une maquette par impression 3D (fig. 14) ; 3) une présentation virtuelle par réalité augmentée. Les tubes accélérateurs disparus pourront ainsi être reconstitués sous forme virtuelle, ainsi que la cage de Faraday et les différents appareillages. En parallèle, une exposition a été réalisée avec différents panneaux explicatifs (biographie de l'objet, enjeux, Jean Thibaud, accélérateur de particules et physique nucléaire, patrimoine et conservation, imaginaire de l'ère de l'atome ${ }^{32}$. 
Figure 13

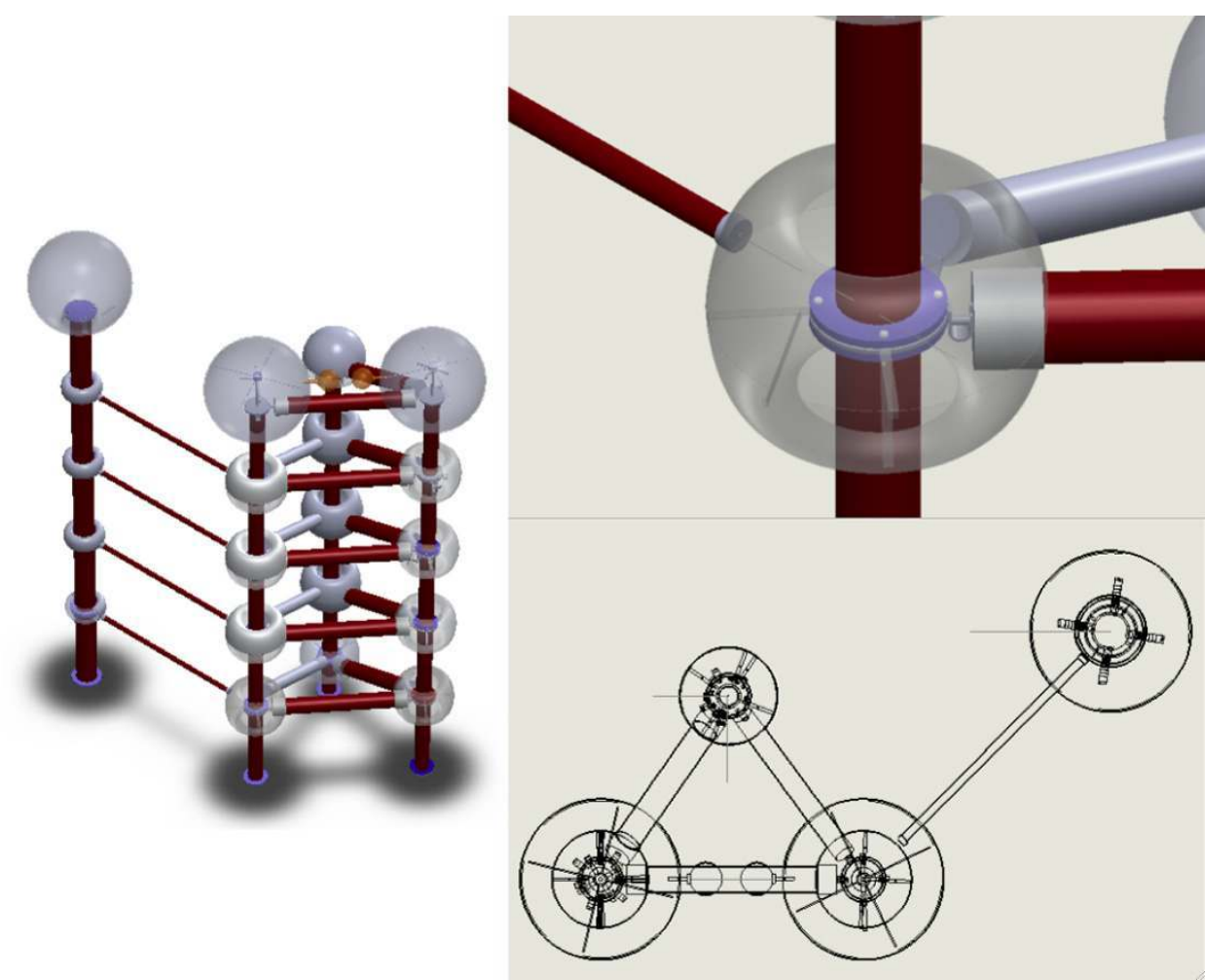

Modélisation 3D du générateur Cockcroft-Walton (vue générale ; vue des éléments de montage ; vue de dessus en esquisse) réalisée par Rémy Geindreau et Philippe Pirello à l'aide du logiciel Solidworks ${ }^{\circledR}$

Mod. Geindreau, R., Pirello, P. @ École supérieure d'Art d'Avignon, 2014 


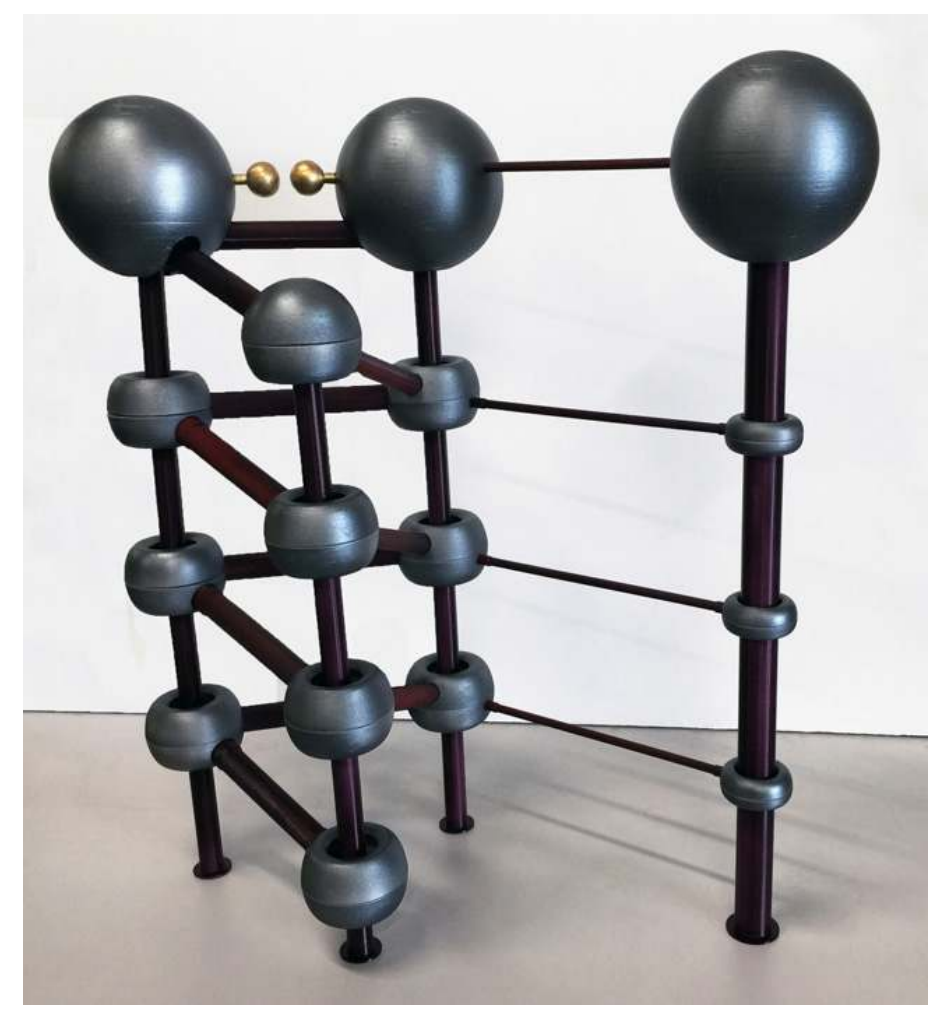

Impression 3D d'une maquette du générateur Cockcroft-Walton par synthèse additive réalisée par Damien Jacques, Groupe Conception Production, INSA de Lyon.

Phot. Panczer, Gérard. ( ) Institut national des sciences appliquées de Lyon, 2016.

\section{En conclusion}

La tâche du technologue, nous dit Simondon, est d'être le représentant des êtres techniques auprès de ceux par qui s'élabore la culture. Aussi, les acteurs du projet d'exposition du Cockcroft-Walton sont-ils des technologues, et afin ne pas galvauder ce terme, nous nous devons de porter une réflexion sur le générateur qui ne le réduise pas à un de ses aspects. Comment, à travers la médiation, diminuer l'écart entre les usages passés de l'objet et ses usages futurs ? Le générateur a toujours été adapté à son milieu et nous avons toutes les bonnes raisons de penser qu'en faire un "objet fermé ", serait dommageable ${ }^{33}$. En conséquence, l'adaptation de l'objet, son ouverture à un nouveau contexte culturel passe, entre autres, par le choix d'un lieu d'exposition permettant non seulement des conditions de conservation préventive adaptée, mais aussi, une présentation de l'objet au plus proche de son milieu d'usage passé (choix du lieu de présentation, modélisation 3D, panneaux didactiques, objets annexes). En quelque sorte, le script ${ }^{34}$ inscrit dans le générateur, sa trajectoire et son inscription complexe dans le temps - qui ne peuvent être confondus à sa stricte ustensilité -, soit celui de participer à la fabrication des sciences dans un laboratoire de physique, doit être transmué dans un autre contexte. Si naguère il permettait de briser les atomes, il va désormais servir à briser la barrière qui sépare encore le monde de l'instrumentation et de la recherche, d'un public plus large. 
NOTES

1. - THIBAUD, Jean. «Réalisation à Lyon d'un accélérateur de particules à un million de volts pour l'étude des réactions nucléaires par bombardement ionique et la production de radioisotopes ». Bulletin du Groupe Sud-Est de la Société française des Électriciens, 1954, p. 13.

2. - BRISSAUD, Yvan, BARON, Éric. «La course des accélérateurs de particules vers les hautes énergies et la Log Périodicité » [document électronique]. Cybergeo: European Journal of Geography, 2007, http://cybergeo.revues.org/14173 [date de consultation 29/01/2015].

3. - CERN (Conseil européen pour la recherche nucléaire) : Organisation européenne pour la recherche nucléaire.

4. - Voir dans la base Palissy : notice PM94000384.

5. - Un générateur de type Cockcroft-Walton est un multiplicateur et redresseur de tension. À partir d'une tension alternative relativement faible, il produit une tension continue élevée en accumulant des charges électriques grâce à une série de condensateurs et de diodes montés en cascade. Ce procédé a été inventé par John Cockcroft et Ernest Walton au Cavendish Laboratory de l'université de Cambridge sous la direction d'Ernest Rutherford. Ils obtiennent le prix Nobel de physique (1951) pour ce montage avec lequel ils réalisent, en 1932, la première transmutation contrôlée de l'histoire ainsi que la première validation expérimentale de la théorie de «l'effet tunnel » de George Gamow et de l'équation d'Albert Einstein « $\mathrm{E}=\mathrm{mc}^{2} »$. Cela dit, ce type de montage est le fruit d'inventeurs successifs à la même époque dans le monde ; on peut noter, par exemple, Heinrich Greinacher à Zurich.

6. - L'Institut de physique nucléaire de Lyon (IPNL) est aujourd'hui une unité mixte de recherche dédiée à l'étude des propriétés des composants subatomiques de la matière ainsi que leurs interactions ions/agrégats-matière...). L'IPNL est sous la tutelle de l'université Claude Bernard Lyon 1 et de l'Institut national de physique nucléaire et de physique des particules (IN2P3) du CNRS.

7. - GEINDREAU, Rémy. Contribution de la conservation-restauration au destin patrimonial d'un générateur Cockcroft-Walton. Avignon : mémoire de fin d'études, école supérieure d'art d'Avignon, 2014.

8. - PESTRE, Dominique. «Les physiciens dans les sociétés occidentales de l'après-guerre. Une mutation des pratiques techniques et des comportements sociaux et culturels». Revue d'histoire moderne et contemporaine, 1992, 39-1, p. 56-72.

9. - DAVAL, Marion. «Les substances radioactives dans les objets patrimoniaux » [document électronique]. CeROArt, $2014<$ http://ceroart.revues.org/4160> [date de consultation 30/01/2015].

10. - RABARDEL, Pierre. Les hommes et les technologies, une approche cognitive des instruments contemporains. Paris : Armand Colin, 1995.

11. - MUMFORD, Lewis. Technique et Civilisation. Paris : éd. du Seuil, 1950.

12. - ECO, Umberto. "Observation sur la notion de gisement culturel ». Traverses, 1993, n5, p. 9.

13. - Bing est une série télévisée réalisée par Nino Monti avec l'aide des équipes de FR3 Lyon et diffusée en 4 épisodes entre 1991 et 1992. Voir SCHLERET, Jean-Jacques, BADOU, Jacques. Merveilleux, fantastique et science-fiction à la télévision française. Bry-sur-Marne : INA et 8eme art édition, 1995.

14. - LEROI-GOURHAN, André. Les racines du monde. Paris : Belfond, 1982, p. 272.

15. - BOURDIEU, Pierre. Science de la science et réflexivité. Paris : Raisons d'agir, 2001, p. 83. 
16. - On peut se référer au travail de thèse en cours de Pascal Bellanca-Penel (S2HEP, Lyon) concernant l'histoire de la physique nucléaire en France de 1920 à 1960 à travers le parcours du physicien Jean Thibaud.

17. - MÉTRAUX, Auguste-Francis. «Accélérateur de protons 1200000 V». Bulletin du Groupe SudEst de la Société française des Électriciens, 1954, p. 14.

18. - THIBAUD, Jean, VERZAUX, P., SALIN, Raymond. «Premiers résultats obtenus avec l'accélérateur de particules à 1 million de volts, installé à Lyon ». Comptes rendus hebdomadaires des séances de l'Académie des sciences, 1954, 239, nº 22.

19. - SALIN, Raymond. «Jean Thibaud, le fondateur ». Dans CHAMBON, Bernard, et al. Institut de physique nucléaire de Lyon. Chronique de 40 années. Lyon : IPNL, 2003, p. 74-78.

20. - ROUX, Jean, MARTIN, Jean. «Construction d'un hall hémisphérique pour essais très haute tension à l'Institut de physique nucléaire de Lyon ». Cahier de recherche interne, LYCEN/6943, IPNL, 1969.

21. - « Préinjecteur de 1,4 MeV ». Courrier CERN, 1970, 10, 3.

22. - MARTIN, Jean. « Le Dôme du Haefely au RFQ ». Dans CHAMBON, Bernard, et al., op. cit.

23. - MARIOT-LEDUC, Sophie. Le patrimoine technique : enjeux et perspectives d'un patrimoine en cours de constitution. Science de l'information et de la Communication. Avignon : université d'Avignon et des pays de Vaucluse, 2008.

24. - AKRICH, Madeleine. "La construction d'un système socio-technique, esquisse pour une anthropologie des techniques ». Anthropologie et Sociétés, 1989, 13, 2, p. 31-54.

25. - La thèse de Séverine Derolez (en cours de rédaction) est consacrée à l'étude des conditions de patrimonialisation et de valorisation du patrimoine scientifique contemporain de la recherche avec le cas du générateur Cockcroft-Walton. Université Lyon 1, Laboratoire S2HEP, Musée des Confluences.

26. - ROLLAND-VILLEMOT, Bénédicte. "Le traitement des collections industrielles et techniques, de la connaissance à la diffusion ». La Lettre de l'OCIM, 2001, 73, p. 13-18.

27. - GRANATO, Marcus, LEGUET-TULLY, Françoise. «Les principes de la restauration d'instruments scientifiques: le cas du cercle méridien Gautier de l'observatoire de Rio de Janeiro" [document électronique]. In Situ, $2009<\mathrm{http}$ ://insitu.revues.org/3865> [date de consultation 30/01/2015].

28. - Certains d'entre eux peuvent parfois être remis en fonction à des fins pédagogiques et démonstratives. C'est le cas du projet de conservation "Montrer l'invisible» soutenu par l'université Claude Bernard et l'IPNL. La restauration/réparation d'une "chambre à étincelles » qui rend perceptible les rayons cosmiques. Les travaux importants (réparation du cœur du détecteur, amélioration des circuits électroniques et révision de la sécurité) sont réalisés par des spécialistes de ce type d'instrument (LPSC de Grenoble).

29. - MUÑOZ VIÑAS, Salvador. Contemporary Theory of Conservation. Oxford/Burlington : Elsevier, 2005. Dans le même sens, voir également l'article: LEVEAU, Pierre. «Les dilemmes philosophiques de la conservation-restauration du patrimoine ». E-conservation magazine, 2009, 12, p. 47-57.

30. - MAIROT, Philippe. «Musée et technique » [document électronique]. Terrain, 16, 1991, < http://terrain.revues.org/3004> [date de consultation 30/01/2015].

31. - DEROLEZ, Séverine, KHANTINE-LANGLOIS, Françoise, LAUTESSE, Philippe. «Le patrimoine scientifique comme ressource pour l'enseignement ». Cahiers de la recherche et du développement, actes des $8^{e s}$ rencontres scientifiques de l'ARDIST, 2014, p. 173-184.

32. - « La Big Science à Lyon : l'accélérateur Cockcroft-Walton ». Exposition, du 31 mai au 2 juillet 2016, Bibliothèque Universitaire des sciences du Campus, Villeurbanne.

33. - GEINDREAU, Rémy. «Défragmenter la technique. Le générateur Cockcroft-Walton du Professeur Thibaud ». Magazine des Cultures digitales MCD. Archéologie des média, 2014, 75, p. 70-73.

34. - SIMONDON, Gilbert. Du mode d'existence des objets techniques. Paris : Aubier, 1958. 


\section{RÉSUMÉS}

Le générateur de haute tension de type Cockcroft-Walton, qui alimentait un accélérateur de particules, est aujourd'hui au cœur d'un projet de restauration et de valorisation. Le travail pluridisciplinaire qui a été engagé propose une étude de faisabilité du projet comprenant une réflexion sur le sens et l'impact du choix du lieu de conservation de cet ensemble technicoscientifique, notre but étant de faciliter la transplantation de l'objet entre ses différents environnements d'usage. Nous proposons d'expliquer les étapes de cette enquête historique et ethno-technologique autour de ce vestige de la « Big Science » en cours de patrimonialisation.

The Cockcroft-Walton high voltage generator, which provided energy for a particle accelerator, is today at the heart of a restoration and interpretation project. The multidisciplinary approach that was involved studied the feasibility of the project, including reflection on the meaning and impact of the place chosen to preserve this scientific and technological ensemble. The objective is to facilitate the relocation of the object between the various contexts of its uses. Our article gives an account of the steps of this historic and ethno-technological investigation around a vestige of 'Big Science', a realm now becoming heritage.

\section{INDEX}

Mots-clés : conservation-restauration, patrimoine technique et scientifique contemporain, physique nucléaire, accélérateur de particules, générateur Cockcroft-Walton

Keywords : conservation-restoration, contemporary technical and scientific heritage, nuclear physics, particle accelerator, Cockcroft-Walton generator

\section{AUTEURS}

\section{RÉMY GEINDREAU}

Conservateur-restaurateur des biens culturels, spécialisé en patrimoine technique, scientifique et industriel. Diplômé de l'école supérieure d'art d'Avignon, département conservation-

restauration remy.geindreau@gmail.com

\section{SÉVERINE DEROLEZ}

Laboratoire Sciences et Société, Historicité, Éducation et Pratiques, université Lyon 1 et musée des Confluences, doctorante CIFRE severine.derolez@gmail.com

\section{JEAN-PAUL MARTIN}

Physicien, Institut de physique nucléaire de Lyon/CNRS martinjp@in2p3.fr 